\title{
ON A GENERALIZATION OF ANDO'S DILATION THEOREM
}

\author{
NIRUPAMA MALLICK AND K. SUMESH
}

\begin{abstract}
We introduce the notion of $Q$-commuting operators which includes commuting operators. We prove a generalized version of the commutant lifting theorem and Ando's dilation theorem in the context of $Q$-commuting operators.
\end{abstract}

\section{INTRODUCTION}

Throughout, $\mathcal{H}$ and $\mathcal{K}$ denote complex Hilbert spaces and $\mathcal{B}(\cdot)$ denotes the space of all bounded linear maps. Suppose $\mathcal{H}_{i}$ and $\mathcal{K}_{i}$ are Hilbert spaces such that $\mathcal{H}_{i} \subseteq \mathcal{K}_{i}, i=1,2$. Given $T \in \mathcal{B}\left(\mathcal{H}_{1}, \mathcal{H}_{2}\right)$ a bounded linear operator $S \in \mathcal{B}\left(\mathcal{K}_{1}, \mathcal{K}_{2}\right)$ is said to be an

(i) extension of $T$ if $S(h)=T(h)$ for all $h \in \mathcal{H}_{1}$. (In such cases we write $\left.S\right|_{\mathcal{H}_{1}}=T$.)

(ii) lifting of $T$ if $S\left(\mathcal{H}_{1}^{\perp}\right) \subseteq \mathcal{H}_{2}^{\perp}$ and $T=\left.P_{\mathcal{H}_{2}} S\right|_{\mathcal{H}_{1}}$ (equivalently $\left.S^{*}\right|_{\mathcal{H}_{2}}=T^{*}$ ).

With respect to the decompositions $\mathcal{K}_{1}=\mathcal{H}_{1} \oplus \mathcal{H}_{1}^{\perp}$ and $\mathcal{K}_{2}=\mathcal{H}_{2} \oplus \mathcal{H}_{2}^{\perp}$, in $(i)$ and (ii) the

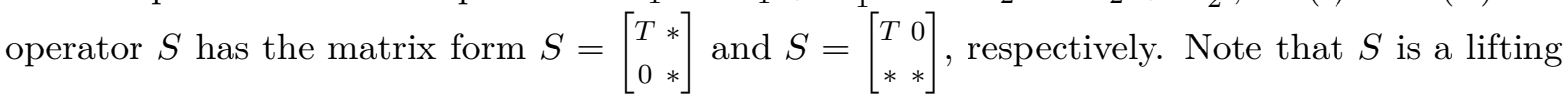
of $T$ if and only if $S^{*}$ is an extension of $T^{*}$.

An operator $S \in \mathcal{B}(\mathcal{K})$ is said to be a dilation of $T \in \mathcal{B}(\mathcal{H})$ if $\mathcal{H} \subseteq \mathcal{K}$ and $T^{n}=\left.P_{\mathcal{H}} S^{n}\right|_{\mathcal{H}}$ for all $n \geq 0$, where $P_{\mathcal{H}} \in \mathcal{B}(\mathcal{K})$ is the orthogonal projection onto $\mathcal{H}$. In such case, with respect to the decomposition $\mathcal{K}=\mathcal{H} \oplus \mathcal{H}^{\perp}$, the operator $S^{n}$ has the matrix form $S^{n}=\left[\begin{array}{c}T^{n} * \\ * *\end{array}\right]$ for all $n \geq 0$. Clearly extension and lifting of $T \in \mathcal{B}(\mathcal{H})$ are dilations. It is well known that (see [6, 7]) given any contraction $T \in \mathcal{B}(\mathcal{H})$ there exists a Hilbert space $\mathcal{K} \supseteq \mathcal{H}$ and an isometry $V \in \mathcal{B}(\mathcal{K})$ such that $V$ is a dilation of $T$. Such a pair $(V, \mathcal{K})$ is called an isometric dilation of $T$. An isometric dilation $(V, \mathcal{K})$ is said to be minimal if

$$
\mathcal{K}=\overline{\operatorname{span}}\left\{V^{n}(\mathcal{H}): n \geq 0\right\} .
$$

Minimal isometric dilations are unique upto unitary equivalence in the sense that if $(V, \mathcal{K})$ and $\left(V^{\prime}, \mathcal{K}^{\prime}\right)$ are two minimal isometric dilations, then there exists a unitary $U: \mathcal{K} \rightarrow \mathcal{K}^{\prime}$ such that $\left.U\right|_{\mathcal{H}}=I_{\mathcal{H}}$ and $U V=V^{\prime} U$. Sz-Nagy proved $([5$, , 7]) that given a contraction $T \in \mathcal{B}(\mathcal{H})$ there exists a Hilbert space $\mathcal{K}$ and a unitary $U \in \mathcal{B}(\mathcal{K})$ such that $U$ is a dilation of $T$. Such a pair $(U, \mathcal{K})$ is called a unitary dilation of $T$. Moreover, such a dilation is unique (up to unitary equivalence) if it is minimal, in the sense that

$$
\mathcal{K}=\overline{\operatorname{span}}\left\{U^{n}(\mathcal{H}): n \in \mathbb{Z}\right\} .
$$

Date: October 31, 2019

2010 Mathematics Subject Classification. 47A20.

Key words and phrases. Isometric dilation, unitary dilation, co-isometric extension, commutant lifting, Ando's dilation theorem.

Nirupama Mallick, Chennai Mathematical Institute, H1, SIPCOT IT Park, Kelambakkam, Siruseri, Tamilnadu603103, India. Email: niru.mallick@gmail.com.

K. Sumesh, Indian Institute of Technology Madras, Sardar Patel Road, Opposite to C. L.R.I, Adyar, Chennai600036, India. Email: sumeshkpl@gmail.com, sumeshkpl@iitm.ac.in. 
In [9] Schaffer gave an elementary proof of the existence of minimal unitary dilation of a contraction.

Given a contraction $T \in \mathcal{B}(\mathcal{H})$ there always exists a Hilbert space $\mathcal{K} \supseteq \mathcal{H}$ and a co-isometry $W \in \mathcal{B}(\mathcal{K})$ which extends $T$, and we call $(W, \mathcal{K})$ a co-isometric extension of $T$. In fact, by considering the lower right-hand corner of the matrix form of the Schaffer's construction (see [9, 7]), one can get a co-isometric extension $(W, \mathcal{K})$ of $T$ which is minimal in the sense that

$$
\mathcal{K}=\overline{\operatorname{span}}\left\{W^{* n}(\mathcal{H}): n \geq 0\right\} .
$$

Note that if $\left(V^{*}, \mathcal{K}\right)$ is a minimal co-isometric extension of $T^{*}$, then $(V, \mathcal{K})$ is an isometric lifting and hence a dilation of $T$ which is minimal in the sense that (1.1) holds. Now from the uniqueness property, it follows that $\mathcal{H}^{\perp}$ is invariant for every minimal isometric dilation of $T \in \mathcal{B}(\mathcal{H})$. Thus, a pair $(V, \mathcal{K})$ is a minimal isometric dilation of $T$ if and only if $(V, \mathcal{K})$ is a minimal isometric lifting of $T$ if and only if $\left(V^{*}, \mathcal{K}\right)$ is a minimal co-isometric extension of $T^{*}$.

Ando ([1]) proved that given any two contractions $T_{1}, T_{2} \in \mathcal{B}(\mathcal{H})$ which are commuting (i.e., $\left.T_{1} T_{2}=T_{2} T_{1}\right)$ there exists a Hilbert space $\mathcal{K}_{0} \supseteq \mathcal{H}$ and commuting isometries $V_{1}, V_{2} \in \mathcal{B}\left(\mathcal{K}_{0}\right)$ such that

$$
T_{1}^{n} T_{2}^{m}=\left.P_{\mathcal{H}} V_{1}^{n} V_{2}^{m}\right|_{\mathcal{H}}
$$

for all $n, m \geq 0$. In fact, $V_{i}$ can be chosen to be a lifting of $T_{i}, i=1,2$. Further, using Ito's theorem [3] he concluded that there exists a Hilbert space $\mathcal{K} \supseteq \mathcal{H}$ and commuting unitary operators $U_{1}, U_{2} \in \mathcal{B}(\mathcal{K})$ such that

$$
T_{1}^{n} T_{2}^{m}=\left.P_{\mathcal{H}} U_{1}^{n} U_{2}^{m}\right|_{\mathcal{H}}
$$

for all $n, m \geq 0$. This is known as the Ando's dilation theorem.

Let $T_{i} \in \mathcal{B}\left(\mathcal{H}_{i}\right)$ be a contraction with isometric lifting $\left(V_{i}, \mathcal{K}_{i}\right)$ (respectively co-isometric extension $\left.\left(W_{i}, \mathcal{K}_{i}\right)\right), i=1,2$. Suppose $X \in \mathcal{B}\left(\mathcal{H}_{1}, \mathcal{H}_{2}\right)$ intertwines $T_{1}$ and $T_{2}$, i.e., $X T_{1}=T_{2} X$. Then, due to Sz-Nagy and Foias ([6], [7]) there exists a norm-preserving lifting (respectively extension) $Y \in \mathcal{B}\left(\mathcal{K}_{1}, \mathcal{K}_{2}\right)$ of $X$ which intertwine $V_{1}$ and $V_{2}$ (respectively $W_{1}$ and $W_{2}$ ). This result is called intertwining lifting (respectively intertwining co-extension) theorem. The case when $T_{1}=T_{2}$ and $V_{1}=V_{2}$ (respectively $W_{1}=W_{2}$ ) is known as commutant lifting (respectively commutant co-extension) theorem.

One may ask how these dilation theorems of commuting pair of contractions can be generalized to the setting of noncommuting pair of contractions $T_{1}, T_{2}$ ? In [8] Sebestyen proved analogues of commutant lifting theorem and Ando's dilation theorem for anticommuting pair (i.e., $\left.T_{2} T_{1}=-T_{1} T_{2}\right)$ of contractions. In [4] Keshari and Mallick considered $q$-commuting operators (i.e., $T_{2} T_{1}=q T_{1} T_{2}$ ) where $q \in \mathbb{T}=\{z \in \mathbb{C}:|z|=1\}$. They proved a generalized version of the commutant lifting theorem, intertwining lifting theorem and Ando's dilation theorem in the context of $q$-commuting contractions, and called them as $q$-commutant lifting theorem, $q$-intertwining lifting theorem and q-commutant dilation theorem respectively. In this article we consider operators $T_{1}$ and $T_{2}$ which are $Q$-commuting i.e., $T_{2} T_{1}$ equals either $Q T_{1} T_{2}, T_{1} Q T_{2}$ or $T_{1} T_{2} Q$ for some bounded operator $Q$. Our main aim is to prove an analogue of the commutant lifting theorem and Ando's dilation theorem to the setting of $Q$-commuting operators. As a first step we characterize (Theorem 2.6) $Q$-commutants of a contraction $T \in \mathcal{B}(\mathcal{H})$ in terms of $\bar{Q}$-commutants of its minimal isometric dilation $(V, \mathcal{K})$, where $\bar{Q}=Q \oplus Q^{\prime} \in \mathcal{B}(\mathcal{K})$ with $Q^{\prime} \in \mathcal{B}\left(\mathcal{H}^{\perp}\right)$. This is a generalized version of $(q$-)commutant lifting theorem $([4,6])$. The 
proof uses Schaffer construction. Further using ideas from [2] we prove generalized versions (see Theorem 2.8, 2.15) of ( $q$-)intertwining lifting theorem. In Theorem 2.14 we characterize $Q$ commutants of a contraction in terms of $\bar{Q}$-commutants of its minimal unitary dilation. Finally, we prove our main theorems that $Q$-commuting contractions can be dilated into $\bar{Q}$-commuting isometries (Theorem 2.16) and further into $\bar{Q}$-commuting unitaries (Theorem 2.20 ). These results generalize Ando's dilation theorem and $q$-commutant dilation theorem. The proofs consist of standard dilation theoretic arguments.

\section{MAIN RESUlts}

Suppose $\mathcal{H}$ and $\mathcal{K}$ are Hilbert spaces such that $\mathcal{H} \subseteq \mathcal{K}$. Given any $Q \in \mathcal{B}\left(\mathcal{H}\right.$ ) we let $\bar{Q}_{\mathcal{K}}$ (or simply $\bar{Q}$ ) denotes any bounded operator on $\mathcal{K}$ such that $\mathcal{H}$ is a reducing subspace for $\bar{Q}$ and $\left.\bar{Q}\right|_{\mathcal{H}}=Q$. Note that $\left(Q \oplus q I_{\mathcal{H}^{\perp}}\right) \in \mathcal{B}(\mathcal{K})$ is an example for such an operator $\bar{Q}$ for every $q \in \mathbb{C}$. If $Q$ is a contraction or (co-)isometry or unitary, then we require $\bar{Q}$ also to be a contraction or (co-)isometry or unitary, respectively.

Definition 2.1. Given $Q \in \mathcal{B}(\mathcal{H})$ two operators $T_{1}, T_{2} \in \mathcal{B}(\mathcal{H})$ are said to be $Q$-commuting if one of the following happens:

$$
T_{2} T_{1}=Q T_{1} T_{2} \text { or } T_{2} T_{1}=T_{1} Q T_{2} \text { or } T_{2} T_{1}=T_{1} T_{2} Q .
$$

If $Q=I_{\mathcal{H}}$ (respectively $Q=-I_{\mathcal{H}}$ ), then $Q$-commuting means commuting (respectively anticommuting).

Example 2.2. Let $T_{1}=\left[\begin{array}{ll}0 & 0 \\ 1 & 1\end{array}\right]$ and $T_{2}=\left[\begin{array}{rr}-2 & 0 \\ 1 & 1\end{array}\right]$ in $M_{2}(\mathbb{C})$. Note that $T_{1}, T_{2}$ are not commuting. In fact, there does not exists any $q \in \mathbb{C}$ such that $T_{2} T_{1}=q T_{1} T_{2}$. But $Q=\left[\begin{array}{cc}-1 & 0 \\ 0 & 1\end{array}\right]$ and $Q^{\prime}=\left[\begin{array}{ll}0 & 0 \\ 0 & 1\end{array}\right]$ in $M_{2}(\mathbb{C})$ are such that $T_{2} T_{1}=T_{1} T_{2} Q=T_{1} Q^{\prime} T_{2}$. Note that there is no $Q \in M_{2}(\mathbb{C})$ such that $T_{2} T_{1}=Q T_{1} T_{2}$

Example 2.3. Suppose $L, R, Q, Q^{\prime} \in \mathcal{B}\left(\ell^{2}\right)$ are the linear operators given by

$$
\begin{aligned}
L\left(x_{1}, x_{2}, x_{3}, \cdots\right) & :=\left(x_{2}, x_{3}, x_{4}, \cdots\right) \\
R\left(x_{1}, x_{2}, x_{3}, \cdots\right) & :=\left(0, x_{1}, x_{2}, x_{3}, \cdots\right) \\
Q\left(x_{1}, x_{2}, x_{3}, \cdots\right) & :=\left(0, x_{2}, x_{3}, x_{4}, \cdots\right) \\
Q^{\prime}\left(x_{1}, x_{2}, x_{3}, \cdots\right) & :=\left(0,0, x_{3}, x_{4}, \cdots\right)
\end{aligned}
$$

Clearly $q L R \neq R L$ for all $q \in \mathbb{C}$. Note that $R L=Q L R=L Q^{\prime} R=L R Q$.

Example 2.4. Suppose $T_{1}=\left[\begin{array}{ll}0 & 1 \\ 0 & 0\end{array}\right]$ and $T_{2}=\left[\begin{array}{ll}0 & 0 \\ 2 & 0\end{array}\right]$ in $M_{2}(\mathbb{C})$. Note that there does not exists any $Q \in M_{2}(\mathbb{C})$ such that $T_{1}$ and $T_{2}$ are $Q$-commuting.

The above example shows that given two operators $S, T \in \mathcal{B}(\mathcal{H})$, there may not exist always an operator $Q \in \mathcal{B}(\mathcal{H})$ such that $S$ and $T$ are $Q$-commuting. However, the next Lemma says that given two operators $T, Q \in \mathcal{B}(\mathcal{H})$, under some suitable conditions, there always exists an 
operator $S \in \mathcal{B}(\mathcal{H})$ such that $S$ and $T$ are $Q$-commuting. This is a generalization of [4, Lemma 3.5]. Recall that $T \in \mathcal{B}(\mathcal{H})$ is called a pure co-isometry if $T T^{*}=I$ and $T^{* n} \rightarrow 0$ in the strong operator topology.

Lemma 2.5. Suppose $T \in \mathcal{B}(\mathcal{H})$ is a pure co-isometry and $Q \in \mathcal{B}(\mathcal{H})$ is an isometry. If $T^{*}(\mathcal{H})$ is invariant for $Q$, then there exists a co-isometry $S \in \mathcal{B}(\mathcal{H})$ such that $T S=S T Q$.

Proof. Since $T^{*}$ is an isometry $\mathcal{W}=\left(T^{*} \mathcal{H}\right)^{\perp}$ is a wandering subspace for $T^{*}$, i.e., $T^{* m}(\mathcal{W}) \perp$ $T^{* n}(\mathcal{W})$ for all $m \neq n \in \mathbb{N}$. Moreover, since $T$ is pure co-isometry $\mathcal{H}=\bigoplus_{n=0}^{\infty} T^{* n}(\mathcal{W})$. As $T^{*}(\mathcal{H}) \subseteq \mathcal{W}^{\perp}$ and $Q^{*}(\mathcal{W}) \subseteq \mathcal{W}$, for $m<n$ and $w, w^{\prime} \in \mathcal{W}$ we have

$$
\left\langle\left(Q T^{*}\right)^{n} w,\left(Q T^{*}\right)^{m} w^{\prime}\right\rangle=\left\langle\left(Q T^{*}\right)^{n-m} w, w^{\prime}\right\rangle=\left\langle T^{*}\left(Q T^{*}\right)^{n-m-1} w, Q^{*} w^{\prime}\right\rangle=0 .
$$

Thus $\left(Q T^{*}\right)^{m}(\mathcal{W}) \perp\left(Q T^{*}\right)^{n}(\mathcal{W})$ for all $m \neq n \in \mathbb{N} \cup\{0\}$. Define $S_{0}: \mathcal{H} \rightarrow \mathcal{H}$ by

$$
S_{0}\left(\sum_{n=0}^{\infty} T^{* n} w_{n}\right)=\sum_{n=0}^{\infty}\left(Q T^{*}\right)^{n+1} w_{n}
$$

Then,

$$
\begin{aligned}
\left\|S_{0}\left(\sum_{n \geq 0} T^{* n} w_{n}\right)\right\|^{2} & =\sum_{n \geq 0} \sum_{m \geq 0}\left\langle\left(Q T^{*}\right)^{n+1} w_{n},\left(Q T^{*}\right)^{m+1} w_{m}\right\rangle \\
& =\sum_{n \geq 0}\left\langle\left(Q T^{*}\right)^{n+1} w_{n},\left(Q T^{*}\right)^{n+1} w_{n}\right\rangle \\
& =\sum_{n \geq 0}\left\langle w_{n}, w_{n}\right\rangle \\
& =\sum_{n \geq 0}\left\langle T^{* n} w_{n}, T^{* n} w_{n}\right\rangle \\
& =\sum_{n \geq 0} \sum_{m \geq 0}\left\langle T^{* n} w_{n}, T^{* m} w_{m}\right\rangle \\
& =\left\|\sum_{n \geq 0} T^{* n} w_{n}\right\|^{2} .
\end{aligned}
$$

Thus $S_{0}$ is a well-defined isometry. Moreover, for $w_{n} \in \mathcal{W}, n \geq 1$ we have

$$
S_{0} T^{*}\left(\sum_{n \geq 0} T^{* n} w_{n}\right)=\sum_{n \geq 0}\left(Q T^{*}\right)^{n+2} w_{n}=Q T^{*} S_{0}\left(\sum_{n \geq 0} T^{* n} w_{n}\right),
$$

hence $S_{0} T^{*}=Q T^{*} S_{0}$. Take adjoint on both sides to get $T S=S T Q$, where $S=S_{0}^{*}$.

2.1. Lifting theorems. We recall some basic facts which we will be using frequently. Suppose $T \in \mathcal{B}(\mathcal{H})$ is a contraction with dilation $(V, \mathcal{K})$ and let $Y \in \mathcal{B}(\mathcal{K})$ be an extension of $X \in \mathcal{B}(\mathcal{H})$. Then w.r.t to the decomposition $\mathcal{K}=\mathcal{H} \oplus \mathcal{H}^{\perp}$ we have $Y=\left[\begin{array}{c}X * \\ 0 *\end{array}\right]$ and $V^{n}=\left[\begin{array}{c}T^{n} * \\ * *\end{array}\right]$ for all $n \geq 0$. Note that $V^{n} Y^{m}=\left[\begin{array}{cc}T^{n} X^{m} & * \\ * & *\end{array}\right]$, so that $T^{n} X^{m}=\left.P_{\mathcal{H}} V^{n} Y^{m}\right|_{\mathcal{H}}$ for all $n, m \geq 0$. Similarly if $Y$ is any lifting of $X$, then $X^{n} T^{m}=\left.P_{\mathcal{H}} Y^{n} V^{m}\right|_{\mathcal{H}}$ for all $n, m \geq 0$.

Now we prove an analogue of the $(q$-)commutant lifting theorem for $Q$-commuting operators.

Theorem 2.6 ( $Q$-commutant lifting). Let $T \in \mathcal{B}(\mathcal{H})$ be a contraction with isometric lifting $(V, \mathcal{K})$, and let $X \in \mathcal{B}(\mathcal{H})$. Suppose $Q \in \mathcal{B}(\mathcal{H})$ and $\bar{Q} \in \mathcal{B}(\mathcal{K})$ are contractions. 
(i) If $X T=Q T X$, then there exists a lifting $Y \in \mathcal{B}(\mathcal{K})$ of $X$ such that $Y V=\bar{Q} V Y$.

(ii) If $X T=T Q X$, then there exists a lifting $Y \in \mathcal{B}(\mathcal{K})$ of $X$ such that $Y V=V \bar{Q} Y$. Further assume that $Q$ and $\bar{Q}$ are unitary.

(iii) If $X T=T X Q$, then there exists a lifting $Y \in \mathcal{B}(\mathcal{K})$ of $X$ such that $Y V=V Y \bar{Q}$.

In all cases $T^{n} X^{m}=\left.P_{\mathcal{H}} V^{n} Y^{m}\right|_{\mathcal{H}}$ and $X^{n} T^{m}=\left.P_{\mathcal{H}} Y^{n} V^{m}\right|_{\mathcal{H}}$ for all $n, m \geq 0$. Moreover, $Y$ can be chosen such that $\|Y\|=\|X\|$.

Proof. (i) Set $\widehat{T}=\left[\begin{array}{cc}Q T & 0 \\ 0 & T\end{array}\right]$ and $\widehat{X}=\left[\begin{array}{ll}0 & X \\ 0 & 0\end{array}\right]$ on $\mathcal{H} \oplus \mathcal{H}$. Let $D=\left(I-\bar{Q}^{*} \bar{Q}\right)^{\frac{1}{2}} \in \mathcal{B}(\mathcal{K})$ and $\mathcal{K}_{0}=\overline{\operatorname{ran}}(D V) \subseteq \mathcal{K}$. Let $\widetilde{\mathcal{K}}=\mathcal{K} \oplus\left(\oplus_{1}^{\infty} \mathcal{K}_{0}\right)$. We consider $\mathcal{H} \subseteq \mathcal{K} \subseteq \widetilde{\mathcal{K}}$ through the canonical identification. Now define $\widetilde{V} \in \mathcal{B}(\widetilde{\mathcal{K}})$ by

$$
\widetilde{V}=\left[\begin{array}{ccccc}
\bar{Q} V & 0 & 0 & \ldots & \ldots \\
D V & 0 & 0 & \ldots & \ldots \\
0 & I_{\mathcal{K}_{0}} & 0 & \ldots & \ldots \\
0 & 0 & I_{\mathcal{K}_{0}} & \ldots & \ldots \\
\vdots & \vdots & \vdots & \ddots & \vdots
\end{array}\right] .
$$

Note that $\widetilde{V}$ is an isometry. Also since $\left.\bar{Q}^{*}\right|_{\mathcal{H}}=Q^{*}$ and $\left.V^{*}\right|_{\mathcal{H}}=T^{*}$ we have

$$
\widetilde{V}^{*} h=(\bar{Q} V)^{*} h=V^{*}\left(\bar{Q}^{*} h\right)=T^{*} Q^{*} h=(Q T)^{*} h
$$

for all $h \in \mathcal{H}$, i.e., $\left.\widetilde{V}^{*}\right|_{\mathcal{H}}=(Q T)^{*}$. Thus $\widetilde{V}$ is an isometric lifting of $Q T$. Set $\widehat{V}=\left[\begin{array}{ll}\widetilde{V} & 0 \\ 0 & V\end{array}\right] \in$ $\mathcal{B}(\widetilde{\mathcal{K}} \oplus \mathcal{K})$. Clearly $\widehat{V}$ is an isometric lifting of the contraction $\widehat{T}$. Since $\widehat{T} \widehat{X}=\widehat{X} \widehat{T}$, by commutant lifting theorem there exists $\widehat{Y} \in \mathcal{B}(\widetilde{\mathcal{K}} \oplus \mathcal{K})$ such that $\widehat{V} \widehat{Y}=\widehat{Y} \widehat{V},\left.\widehat{Y}^{*}\right|_{\mathcal{H} \oplus \mathcal{H}}=\widehat{X}^{*}$ and $\|\widehat{Y}\|=$

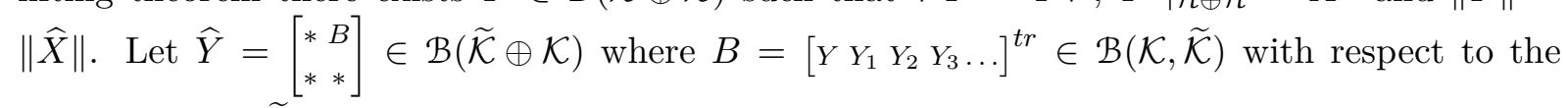
decomposition $\widetilde{\mathcal{K}}=\mathcal{K} \oplus\left(\oplus_{1}^{\infty} \mathcal{K}_{0}\right)$. Then

$$
\widehat{V} \hat{Y}=\widehat{Y} \widehat{V} \Longrightarrow \widetilde{V} B=B V \Longrightarrow \bar{Q} V Y=Y V
$$

where $Y \in \mathcal{B}(\mathcal{K})$. Also

$$
\left.\widehat{Y}^{*}\right|_{\mathcal{H} \oplus \mathcal{H}}=\left.\widehat{X}^{*} \Longrightarrow B^{*}\right|_{\mathcal{H}}=\left.X^{*} \Longrightarrow Y^{*}\right|_{\mathcal{H}}=X^{*},
$$

so that $Y$ is a lifting of $X$. Hence $\|X\| \leq\|Y\| \leq\|B\| \leq\|\widehat{Y}\|=\|\widehat{X}\|=\|X\|$.

(ii) Set $\widehat{T}=\left[\begin{array}{cc}T Q & 0 \\ 0 & T\end{array}\right]$ on $\mathcal{H} \oplus \mathcal{H}$. Let $\widehat{X}, D, \widetilde{\mathcal{K}}$ be as in case (i) with $\mathcal{K}_{0}=\overline{\operatorname{ran}}(V D) \subseteq \mathcal{K}$. Define $\widetilde{V} \in \mathcal{B}(\widetilde{\mathcal{K}})$ by

$$
\widetilde{V}=\left[\begin{array}{ccccc}
V \bar{Q} & 0 & 0 & \ldots & \ldots \\
V D & 0 & 0 & \ldots & \ldots \\
0 & I_{\mathcal{K}_{0}} & 0 & \ldots & \ldots \\
0 & 0 & I_{\mathcal{K}_{0}} & \ldots & \ldots \\
\vdots & \vdots & \vdots & \ddots & \vdots
\end{array}\right] .
$$

Note that $\widehat{V}=\left[\begin{array}{ll}\widetilde{V} & 0 \\ 0 & V\end{array}\right] \in \mathcal{B}(\widetilde{\mathcal{K}} \oplus \mathcal{K})$ is an isometric lifting of the contraction $\widehat{T}$, and since $\widehat{T} \widehat{X}=$ $\widehat{X} \widehat{T}$, by proceeding as in case (i) we can get $Y \in \mathcal{B}(\mathcal{K})$ such that $V \bar{Q} Y=Y V,\left.Y^{*}\right|_{\mathcal{H}}=X^{*}$ and $\|Y\|=\|X\|$. 
(iii) Suppose $Q$ is a unitary. Set $\widehat{T}=\left[\begin{array}{cc}T Q^{*} & 0 \\ 0 & T\end{array}\right]$ and $\widehat{X}=\left[\begin{array}{ll}0 & 0 \\ X & 0\end{array}\right]$ on $\mathcal{H} \oplus \mathcal{H}$, and $\widehat{V}=\left[\begin{array}{cc}V \bar{Q}^{*} & 0 \\ 0 & V\end{array}\right]$ on $\mathcal{K} \oplus \mathcal{K}$. Note that $(\widehat{V}, \mathcal{K} \oplus \mathcal{K})$ is an isometric lifting of $\widehat{T}$. Since $\widehat{T} \widehat{X}=\widehat{X} \widehat{T}$, by commutant lifting theorem there exists a lifting $\widehat{Y}=\left[\begin{array}{c}* * \\ Y *\end{array}\right] \in \mathcal{B}(\mathcal{K} \oplus \mathcal{K})$ of $\widehat{X}$ such that $\widehat{Y} \widehat{V}=\widehat{V} \widehat{Y}$ and $\|\widehat{Y}\|=\|\widehat{X}\|$. Observe that $Y$ is the required lifting of $X$. This completes the proof.

Remark 2.7. In Theorem 2.6(iii) suppose $Q$ is only a co-isometry, so that $T X=X T Q^{*}$. The above proof shows that, in such case also we can get a lifting $Y$ of $X$ satisfying all properties except the equality $V Y \bar{Q}=Y V$, but we get $V Y=Y V \bar{Q}^{*}$.

Theorem 2.8 (Q-intertwining lifting). Let $T_{i} \in \mathcal{B}\left(\mathcal{H}_{i}\right)$ be a contraction with isometric lifting $\left(V_{i}, \mathcal{K}_{i}\right), i=1,2$, and let $X \in \mathcal{B}\left(\mathcal{H}_{1}, \mathcal{H}_{2}\right)$. Suppose $Q \in \mathcal{B}\left(\mathcal{H}_{2}\right)$ and $\bar{Q} \in \mathcal{B}\left(\mathcal{K}_{2}\right)$ are contractions.

(i) If $X T_{1}=Q T_{2} X$, then there exists a lifting $Y \in \mathcal{B}\left(\mathcal{K}_{1}, \mathcal{K}_{2}\right)$ of $X$ such that $Y V_{1}=\bar{Q} V_{2} Y$.

(ii) If $X T_{1}=T_{2} Q X$, then there exists a lifting $Y \in \mathcal{B}\left(\mathcal{K}_{1}, \mathcal{K}_{2}\right)$ of $X$ such that $Y V_{1}=V_{2} \bar{Q} Y$. Suppose $Q \in \mathcal{B}\left(\mathcal{H}_{1}\right)$ and $\bar{Q} \in \mathcal{B}\left(\mathcal{K}_{1}\right)$ are unitary.

(iii) If $X T_{1}=T_{2} X Q$, then there exists a lifting $Y \in \mathcal{B}\left(\mathcal{K}_{1}, \mathcal{K}_{2}\right)$ of $X$ such that $Y V_{1}=V_{2} Y \bar{Q}$. In all cases $T_{2}^{n} X=\left.P_{\mathcal{H}_{2}} V_{2}^{n} Y\right|_{\mathcal{H}_{1}}$ and $X T_{1}^{n}=\left.P_{\mathcal{H}_{2}} Y V_{1}^{n}\right|_{\mathcal{H}_{1}}$ for all $n \geq 0$. Moreover, $Y$ can be chosen such that $\|Y\|=\|X\|$.

Proof. First assume that $X T_{1}=Q T_{2} X$. Set

$$
\begin{aligned}
& \widehat{T}=\left[\begin{array}{cc}
T_{1} & 0 \\
0 & T_{2}
\end{array}\right], \widehat{X}=\left[\begin{array}{cc}
0 & 0 \\
X & 0
\end{array}\right], \mathcal{Q}=\left[\begin{array}{cc}
I_{\mathcal{H}_{1}} & 0 \\
0 & Q
\end{array}\right] \in \mathcal{B}\left(\mathcal{H}_{1} \oplus \mathcal{H}_{2}\right), \quad \text { and } \\
& \overline{\mathcal{Q}}=\left[\begin{array}{cc}
I_{\mathcal{K}_{1}} & 0 \\
0 & \bar{Q}
\end{array}\right], \widehat{V}=\left[\begin{array}{cc}
V_{1} & 0 \\
0 & V_{2}
\end{array}\right] \in \mathcal{B}\left(\mathcal{K}_{1} \oplus \mathcal{K}_{2}\right) .
\end{aligned}
$$

Note that $\mathcal{H}_{1} \oplus \mathcal{H}_{2}$ is reducing for the contraction $\overline{\mathcal{Q}}$, and $\left.\overline{\mathcal{Q}}\right|_{\mathcal{H}_{1} \oplus \mathcal{H}_{2}}=\mathcal{Q}$. Since $\widehat{X} \widehat{T}=\mathcal{Q} \widehat{T} \widehat{X}$ and $\widehat{V}$ is an isometric lifting of $\widehat{T}$, by Theorem 2.6 there exists a lifting $\widehat{Y}=\left[\begin{array}{c}* * \\ Y *\end{array}\right] \in \mathcal{B}\left(\mathcal{K}_{1} \oplus \mathcal{K}_{2}\right)$ of $\widehat{X}$ such that $\widehat{Y} \widehat{V}=\overline{\mathcal{Q}} \widehat{V} \widehat{Y}$ and $\|\widehat{Y}\|=\|\widehat{X}\|$. As $\widehat{Y} \widehat{V}=\overline{\mathcal{Q}} \widehat{V} \widehat{Y}$ we get $Y V_{1}=\bar{Q} V_{2} Y$. Also since $\left.\widehat{Y}^{*}\right|_{\mathcal{H}_{1} \oplus \mathcal{H}_{2}}=\widehat{X}^{*}$ we have $\left.Y^{*}\right|_{\mathcal{H}_{2}}=X^{*}$, i.e., $Y$ is a lifting of $X$. Hence $\|X\| \leq\|Y\| \leq\|\widehat{Y}\|=$ $\|\widehat{X}\|=\|X\|$. The case when $T_{2} Q X=X T_{1}$ can be proved similarly since $\widehat{T} \mathcal{Q} \widehat{X}=\widehat{X} \widehat{T}$. For the case when $X T_{1}=T_{2} X Q$ repeat the above process by taking $\mathcal{Q}=\left[\begin{array}{cc}Q & 0 \\ 0 & I_{\mathcal{H}_{2}}\end{array}\right] \in \mathcal{B}\left(\mathcal{H}_{1} \oplus \mathcal{H}_{2}\right)$ and $\overline{\mathcal{Q}}=\left[\begin{array}{cc}\bar{Q} & 0 \\ 0 & \overline{\mathcal{K}}_{2}\end{array}\right] \in \mathcal{B}\left(\mathcal{K}_{1} \oplus \mathcal{K}_{2}\right)$

Remark 2.9. Theorem 2.8 can also be deduced from the classical intertwining theorem as follows: To prove $(i)$ of Theorem 2.8 let $(\widetilde{V}, \widetilde{\mathcal{K}})$ be an isometric lifting of the contraction $\bar{Q} V_{2}=\left[\begin{array}{cc}Q T_{2} & 0 \\ * & *\end{array}\right] \epsilon$ $\mathcal{B}\left(\mathcal{K}_{2}\right)$. Then $(\widetilde{V}, \widetilde{\mathcal{K}})$ is also an isometric lifting of $Q T_{2}$. Since $X T_{1}=\left(Q T_{2}\right) X$, the classical intertwining lifting theorem yields a lifting $\widehat{Y} \in \mathcal{B}\left(\mathcal{K}_{1}, \widetilde{\mathcal{K}}\right)$ of $X$ such that $\widehat{Y} V_{1}=\widetilde{V} \widehat{Y}$ and $\|\widehat{Y}\|=\|X\|$. With respect to the decomposition $\widetilde{\mathcal{K}}=\mathcal{K}_{2} \oplus \mathcal{K}_{2}^{\perp}$ let $\widetilde{V}=\left[\begin{array}{cc}\bar{Q} V_{2} & 0 \\ * & *\end{array}\right]$ and $\widehat{Y}=\left[\begin{array}{c}Y \\ Y_{0}\end{array}\right]$ with $Y \in \mathcal{B}\left(\mathcal{K}_{1}, \mathcal{K}_{2}\right)$. Then $\widehat{Y} V_{1}=\tilde{V} \widehat{Y}$ implies that $Y V_{1}=\bar{Q} V_{2} Y$. Also, since $\widehat{Y}$ is a lifting of $X$ we have $Y$ is a lifting of $X$, so that $\|X\| \leq\|Y\| \leq\|\widehat{Y}\|=\|X\|$. Part (ii) is proved similarly by replacing $\bar{Q} V_{2}$ with $V_{2} \bar{Q}$. For $(i i i)$, rewrite the assumption as $X\left(T_{1} Q^{*}\right)=T_{2} X$, and note 
that $V_{1} \bar{Q}^{*}$ is already an isometric lifting of $T_{1} Q^{*}$, so the result follows even more directly from classical intertwining lifting theorem. Specializing Theorem 2.8 to $T_{1}=T_{2}$ and $V_{1}=V_{2}$, we obtain Theorem 2.6 ,

Suppose $T \in \mathcal{B}(\mathcal{H})$. Recall that $(V, \mathcal{K})$ is an isometric lifting of a $T$ if and only if $\left(V^{*}, \mathcal{K}\right)$ is an co-isometric extension of $T^{*}$. So we can restate the Theorems 2.6, 2.8 as follows. We will be using this versions later.

Theorem 2.10 ( $Q$-commutant extension). Let $T \in \mathcal{B}(\mathcal{H})$ be a contraction with co-isometric extension $(W, \mathcal{K})$, and let $X \in \mathcal{B}(\mathcal{H})$. Suppose $Q \in \mathcal{B}(\mathcal{H})$ and $\bar{Q} \in \mathcal{B}(\mathcal{K})$ are contractions.

(i) If $X T Q=T X$, then there exists an extension $Y \in \mathcal{B}(\mathcal{K})$ of $X$ such that $Y W \bar{Q}=W Y$.

(ii) If $X Q T=T X$, then there exists an extension $Y \in \mathcal{B}(\mathcal{K})$ of $X$ such that $Y \bar{Q} W=W Y$. Further assume that $Q$ and $\bar{Q}$ are unitary.

(iii) If $Q X T=T X$, then there exists an extension $Y \in \mathcal{B}(\mathcal{K})$ of $X$ such that $\bar{Q} Y W=W Y$. In all cases $T^{n} X^{m}=\left.P_{\mathcal{H}} W^{n} Y^{m}\right|_{\mathcal{H}}$ and $X^{n} T^{m}=\left.P_{\mathcal{H}} Y^{n} W^{m}\right|_{\mathcal{H}}$ for all $n, m \geq 0$. Moreover, $Y$ can be chosen such that $\|Y\|=\|X\|$.

Theorem 2.11 (Q-intertwining extension). Let $T_{i} \in \mathcal{B}\left(\mathcal{H}_{i}\right)$ be a contraction with co-isometric extension $\left(W_{i}, \mathcal{K}_{i}\right), \quad i=1,2$ and let $X \in \mathcal{B}\left(\mathcal{H}_{1}, \mathcal{H}_{2}\right)$. Suppose $Q \in \mathcal{B}\left(\mathcal{H}_{1}\right)$ and $\bar{Q} \in \mathcal{B}\left(\mathcal{K}_{1}\right)$ are contractions.

(i) If $X T_{1} Q=T_{2} X$, then there exists an extension $Y \in \mathcal{B}\left(\mathcal{K}_{1}, \mathcal{K}_{2}\right)$ of $X$ such that $Y W_{1} \bar{Q}=$ $W_{2} Y$.

(ii) If $X Q T_{1}=T_{2} X$, then there exists an extension $Y \in \mathcal{B}\left(\mathcal{K}_{1}, \mathcal{K}_{2}\right)$ of $X$ such that $Y \bar{Q} W_{1}=$ $W_{2} Y$.

Suppose $Q \in \mathcal{B}\left(\mathcal{H}_{2}\right)$ and $\bar{Q} \in \mathcal{B}\left(\mathcal{K}_{2}\right)$ are unitary.

(iii) If $Q X T_{1}=T_{2} X$, then there exists an extension $Y \in \mathcal{B}\left(\mathcal{K}_{1}, \mathcal{K}_{2}\right)$ of $X$ such that $\bar{Q} Y W_{1}=$ $W_{2} Y$.

In all cases $T_{2}^{n} X=\left.P_{\mathcal{H}_{2}} V_{2}^{n} Y\right|_{\mathcal{H}_{1}}$ and $X T_{1}^{n}=\left.P_{\mathcal{H}_{2}} Y V_{1}^{n}\right|_{\mathcal{H}_{1}}$ for all $n \geq 0$. Moreover, $Y$ can be chosen such that $\|Y\|=\|X\|$.

Remark 2.12. Note that case $(i)$ is a stronger version of [10, Theorem 3]. In [10] Sebestyen considered $\bar{Q} \in \mathcal{B}\left(\mathcal{K}_{1}\right)$ with the additional assumption that $\overline{\operatorname{span}}\left\{W_{1}^{* k} h: h \in \mathcal{H}_{1}, 0 \leq k \leq n\right\}$ reduces $\bar{Q}$ for every $n \geq 0$.

Recall that the minimal isometric dilation is an isometric lifting. Thus, Theorem 2.6 characterizes the operators $X$ which are $Q$-commutant to $T$ in terms of the operators $Y$ which are $\bar{Q}$-commutant to the minimal isometric dilation $V$ of $T$. Next we characterizes the operators $X$ which are $Q$-commutant to $T$ in terms of the operators $Y$ which are $\bar{Q}$-commutant to the minimal unitary dilation of $T$, provided $Q$ is a unitary. To prove our result we use the following lemma. 
Lemma $2.13([2])$. Suppose $T \in \mathcal{B}(\mathcal{H})$ is a contraction with the unique minimal co-isometric extension $\left(W, \mathcal{K}_{0}\right)$. Let $\left(U^{*}, \mathcal{K}\right)$ be the unique minimal co-isometric extension of $W^{*}$. Then $U^{*}$ is a unitary, and $(U, \mathcal{K})$ is the unique minimal unitary dilation of $T$.

Theorem 2.14. Let $T \in \mathcal{B}(\mathcal{H})$ be a contraction with the minimal unitary dilation $(U, \mathcal{K})$. Suppose $Q \in \mathcal{B}(\mathcal{H})$ is a unitary and let $X \in \mathcal{B}(\mathcal{H})$.

(i) If $X T=Q T X$, then there exist $\bar{Q} \in \mathcal{B}(\mathcal{K})$ unitary and a dilation $Y \in \mathcal{B}(\mathcal{K})$ of $X$ such that $Y U=\bar{Q} U Y$.

(ii) If $X T=T X Q$, then there exist $\bar{Q} \in \mathcal{B}(\mathcal{K})$ unitary and a dilation $Y \in \mathcal{B}(\mathcal{K})$ of $X$ such that $Y U=U Y \bar{Q}$.

In fact, given $q \in \mathbb{T}$ we can choose $\bar{Q}=Q \oplus q I_{\mathcal{H}^{\perp}}$. In all cases $X^{n} T^{m}=\left.P_{\mathcal{H}} Y^{n} U^{m}\right|_{\mathcal{H}}$ and $T^{n} X^{m}=\left.P_{\mathcal{H}} U^{n} Y^{m}\right|_{\mathcal{H}}$ for all $n, m \geq 0$. Moreover, $Y$ can be chosen such that $\|Y\|=\|X\|$.

Proof. We prove only the case $(i)$. Case (ii) can be proved similarly. Suppose $q \in \mathbb{T}$ and $\left(W, \mathcal{K}_{0}\right)$ is the minimal co-isometric extension of $T$. From Lemma 2.13 and the uniqueness of minimal unitary dilation we can assume that $\left(U^{*}, \mathcal{K}\right)$ is the minimal co-isometric extension of $W^{*}$. Note that $\mathcal{H} \subseteq \mathcal{K}_{0} \subseteq \mathcal{K}$. Let $Q_{0}=Q \oplus q I_{\mathcal{K}_{0} \ominus \mathcal{H}} \in \mathcal{B}\left(\mathcal{K}_{0}\right)$. Since $Q^{*} X T=T X$, by Theorem 2.10 (iii) there exists an extension $Y_{0} \in \mathcal{B}\left(\mathcal{K}_{0}\right)$ of $X$ with $\left\|Y_{0}\right\|=\|X\|$ such that $Q_{0}^{*} Y_{0} W=W Y_{0}, T^{n} X^{m}=\left.P_{\mathcal{H}} W^{n} Y_{0}^{m}\right|_{\mathcal{H}}$ and $X^{n} T^{m}=\left.P_{\mathcal{H}} Y_{0}^{n} W^{m}\right|_{\mathcal{H}}$ for all $n, m \geq 0$. Again since $Y_{0}^{*} W^{*} Q_{0}^{*}=W^{*} Y_{0}^{*}$, by Theorem [2.10 (i) there exists an extension $Y^{*} \in \mathcal{B}(\mathcal{K})$ of $Y_{0}^{*}$ with $\left\|Y^{*}\right\|=\left\|Y_{0}^{*}\right\|$ such that $Y^{*} U^{*}\left(Q \oplus q I_{\mathcal{K} \ominus \mathcal{H}}\right)^{*}=U^{*} Y^{*}, W^{* n} Y_{0}^{* m}=P_{\mathcal{K}_{0}} U^{* n} Y^{* m}{\mid \mathcal{K}_{0}}$ and $Y_{0}^{* n} W^{* m}=\left.P_{\mathcal{K}_{0}} Y^{* n} U^{* m}\right|_{\mathcal{K}_{0}}$ for all $n, m \geq 0$. Note that $Y$ has the required properties.

Theorem 2.15. Let $T_{i} \in \mathcal{B}\left(\mathcal{H}_{i}\right)$ be a contraction with the minimal unitary dilation $\left(U_{i}, \mathcal{K}_{i}\right), i=$ 1,2 and $X \in \mathcal{B}\left(\mathcal{H}_{1}, \mathcal{H}_{2}\right)$.

(i) Suppose $Q \in \mathcal{B}\left(\mathcal{H}_{2}\right)$ is a unitary such that $X T_{1}=Q T_{2} X$. Then there exist $\bar{Q} \in \mathcal{B}\left(\mathcal{K}_{2}\right)$ unitary and $Y \in \mathcal{B}\left(\mathcal{K}_{1}, \mathcal{K}_{2}\right)$ such that $Y U_{1}=\bar{Q} U_{2} Y$.

(ii) Suppose $Q \in \mathcal{B}\left(\mathcal{H}_{1}\right)$ is a unitary such that $X T_{1}=T_{2} X Q$. Then there exist $\bar{Q} \in \mathcal{B}(\mathcal{K})$ unitary and $Y \in \mathcal{B}\left(\mathcal{K}_{1}, \mathcal{K}_{2}\right)$ such that $Y U_{1}=U_{2} Y \bar{Q}$.

In fact, given $q \in \mathbb{T}$ we can choose $\bar{Q}=Q \oplus q I$. In all cases $X T_{1}^{n}=\left.P_{\mathcal{H}_{2}} Y U_{1}^{n}\right|_{\mathcal{H}_{1}}$ and $T_{2}^{n} X=$ $\left.P_{\mathcal{H}_{2}} U_{2}^{n} Y\right|_{\mathcal{H}_{1}}$ for all $n \geq 0$. Moreover, $Y$ can be chosen such that $\|Y\|=\|X\|$.

Proof is similar to that of Theorem 2.8

2.2. Dilation theorems. In this section we prove an analogue of Ando's dilation theorem for $Q$-commuting contractions.

Theorem 2.16 ( $Q$-commuting isometric dilation). Let $T_{1}, T_{2} \in \mathcal{B}(\mathcal{H})$ be contractions and $Q \in \mathcal{B}(\mathcal{H})$ be a unitary such that $T_{2} T_{1}=Q T_{1} T_{2}$. Then there exists a Hilbert space $\mathcal{K} \supseteq \mathcal{H}$, isometries $V_{1}, V_{2} \in \mathcal{B}(\mathcal{K})$ and $\bar{Q} \in \mathcal{B}(\mathcal{K})$ unitary such that

(i) $V_{2} V_{1}=\bar{Q} V_{1} V_{2}$; and

(ii) $V_{i}$ is a lifting (and hence a dilation) of $T_{i}$ so that $T_{1}^{n} T_{2}^{m}=\left.P_{\mathcal{H}} V_{1}^{n} V_{2}^{m}\right|_{\mathcal{H}}$ and $T_{2}^{n} T_{1}^{m}=$ $\left.P_{\mathcal{H}} V_{2}^{n} V_{1}^{m}\right|_{\mathcal{H}}$ for all $n, m \geq 0$. 
In fact, given $q \in \mathbb{T}$ we can choose $\bar{Q}=Q \oplus q I_{\mathcal{K} \ominus \mathcal{H}}$.

Proof. Fix $q \in \mathbb{T}$. Let $\left(\widehat{V}_{1}, \widehat{\mathcal{K}}\right)$ be the minimal isometric dilation of $T_{1}$. Since $T_{2} T_{1}=Q T_{1} T_{2}$, by Theorem 2.6(i) there exists $\widehat{V}_{2} \in \mathcal{B}(\widehat{\mathcal{K}})$ such that

$$
\widehat{V}_{2} \widehat{V}_{1}=\left(Q \oplus q I_{\widehat{\mathcal{K}} \ominus \mathcal{H}}\right) \widehat{V}_{1} \widehat{V}_{2},\left.\quad \widehat{V}_{2}^{*}\right|_{\mathcal{H}}=T_{2}^{*} \quad \text { and }\left\|\widehat{V}_{2}\right\|=\left\|T_{2}\right\| \leq 1 .
$$

Suppose $\left(V_{2}, \mathcal{K}\right)$ is the minimal isometric dilation of $\widehat{V}_{2}$. Note that $\mathcal{H} \subseteq \widehat{\mathcal{K}} \subseteq \mathcal{K}$. Since $\widehat{V}_{1} \widehat{V}_{2}=$ $\left(Q \oplus q I_{\widehat{\mathcal{K}} \ominus \mathcal{H}}\right) * \widehat{V}_{2} \widehat{V}_{1}$, from Theorem 2.6(i) we get $V_{1} \in \mathcal{B}(\mathcal{K})$ such that

$$
V_{1} V_{2}=\left(Q \oplus q I_{\mathcal{K} \ominus \mathcal{H}}\right)^{*} V_{2} V_{1},\left.\quad V_{1}^{*}\right|_{\widehat{\mathcal{K}}}=\widehat{V}_{1}^{*} \quad \text { and }\left\|V_{1}\right\|=\left\|\widehat{V}_{1}\right\| \leq 1 .
$$

Let $V_{1}=\left[\begin{array}{ll}\widehat{V}_{1} & 0 \\ A & B\end{array}\right]$ w.r.t the decomposition $\mathcal{K}=\widehat{\mathcal{K}} \oplus \widehat{\mathcal{K}}^{\perp}$. Since

$$
0 \leq \widehat{V}_{1}^{*} \widehat{V}_{1}+A^{*} A \leq\left\|\widehat{V}_{1}^{*} \widehat{V}_{1}+A^{*} A\right\| I \leq\left\|V_{1}^{*} V_{1}\right\| I \leq I
$$

and $\widehat{V}_{1}$ is an isometry we have $A=0$, so that $\left.V_{1}\right|_{\widehat{\mathcal{K}}}=\widehat{V}_{1}$. Since $Q$ and $V_{2}$ are isometries we have

$$
\left\|V_{1} V_{2}^{n} k\right\|=\left\|\left(Q \oplus q I_{\mathcal{H}^{\perp}}\right) V_{1} V_{2}^{n} k\right\|=\left\|V_{2} V_{1} V_{2}^{n-1} k\right\|=\left\|V_{1} V_{2}^{n-1} k\right\| \quad \forall n \geq 1 .
$$

Repeating the above step recursively we get

$$
\left\|V_{1} V_{2}^{n} k\right\|=\left\|V_{1} V_{2} k\right\|=\left\|\left(Q \oplus q I_{\mathcal{H}^{\perp}}\right) V_{1} V_{2} k\right\|=\left\|V_{2} V_{1} k\right\|=\left\|V_{1} k\right\|=\left\|\widehat{V}_{1} k\right\|=\|k\|=\left\|V_{2}^{n} k\right\|
$$

for every $k \in \widehat{\mathcal{K}}$ and $n \geq 1$. Clearly the above equality holds for $n=0$. Hence

$$
\left\|\left(I-V_{1}^{*} V_{1}\right)^{\frac{1}{2}} V_{2}^{n} k\right\|^{2}=\left\langle V_{2}^{n} k, V_{2}^{n} k\right\rangle-\left\langle V_{2}^{n} k, V_{1}^{*} V_{1} V_{2}^{n} k\right\rangle=\left\|V_{2}^{n} k\right\|^{2}-\left\|V_{1} V_{2}^{n} k\right\|^{2}=0
$$

for all $k \in \widehat{\mathcal{K}}$ and $n \geq 0$. Since $\mathcal{K}=\overline{\operatorname{span}}\left\{V_{2}^{n} k: k \in \widehat{\mathcal{K}}, n \geq 0\right\}$ from above equation we get $\left(I-V_{1}^{*} V_{1}\right)=0$, i.e., $V_{1}$ is an isometry. Moreover, since minimal isometric dilations are liftings we have $\left.V_{i}^{*}\right|_{\mathcal{H}}=\left.\left(\left.V_{i}^{*}\right|_{\widehat{\mathcal{K}}}\right)\right|_{\mathcal{H}}=\left.\widehat{V}_{i}^{*}\right|_{\mathcal{H}}=T_{i}^{*}$ for $i=1,2$. Thus $V_{1}, V_{2}$ are isometric lifting of $T_{1}, T_{2}$ respectively, so that (ii) follows. This completes the proof.

Corollary 2.17 (Q-commuting co-isometric dilation). Let $T_{1}, T_{2} \in \mathcal{B}(\mathcal{H})$ be contractions and $Q \in$ $\mathcal{B}(\mathcal{H})$ be a unitary such that $T_{2} T_{1}=T_{1} T_{2} Q$. Then there exists a Hilbert space $\mathcal{K} \supseteq \mathcal{H}$, coisometries $W_{1}, W_{2} \in \mathcal{B}(\mathcal{K})$ and $\bar{Q} \in \mathcal{B}(\mathcal{K})$ unitary such that

(i) $W_{2} W_{1}=W_{1} W_{2} \bar{Q}$; and

(ii) $W_{i}$ is an extension (and hence a dilation) of $T_{i}$ so that $T_{1}^{n} T_{2}^{m}=\left.P_{\mathcal{H}} W_{1}^{n} W_{2}^{m}\right|_{\mathcal{H}}$ and $T_{2}^{n} T_{1}^{m}=\left.P_{\mathcal{H}} W_{2}^{n} W_{1}^{m}\right|_{\mathcal{H}}$ for all $n, m \geq 0$.

In fact, given $q \in \mathbb{T}$ we can choose $\bar{Q}=Q \oplus q I_{\mathcal{K} \ominus \mathcal{H}}$.

Proof. Fix $q \in \mathbb{T}$. Since $T_{1}^{*} T_{2}^{*}=Q^{*} T_{2}^{*} T_{1}^{*}$, by Theorem 2.16 there exists Hilbert space $\mathcal{K} \supseteq \mathcal{H}$ and an isometric lifting $W_{i}^{*} \in \mathcal{B}(\mathcal{K})$ of $T_{i}^{*}$ such that $W_{1}^{*} W_{2}^{*}=\left(Q \oplus q I_{\mathcal{H}^{\perp}}\right)^{*} W_{2}^{*} W_{1}^{*}$. Note that $W_{i} \in \mathcal{B}(\mathcal{K}), i=1,2$ are the required co-isometric extensions.

Theorem 2.18. Let $V_{1}, V_{2} \in \mathcal{B}(\mathcal{H})$ be isometries and $Q \in \mathcal{B}(\mathcal{H})$ be a unitary such that $V_{2} V_{1}=$ $Q V_{1} V_{2}$. Then there exists a Hilbert space $\mathcal{K} \supseteq \mathcal{H}$ and unitaries $\bar{Q}, U_{1}, U_{2} \in \mathcal{B}(\mathcal{K})$ such that

(i) $U_{2} U_{1}=\bar{Q} U_{1} U_{2} ;$ and 
(ii) $U_{i}$ is an extension (and hence a dilation) of $V_{i}$ so that $V_{1}^{n} V_{2}^{m}=\left.P_{\mathcal{H}} U_{1}^{n} U_{2}^{m}\right|_{\mathcal{H}}$ and $V_{2}^{n} V_{1}^{m}=$ $\left.P_{\mathcal{H}} U_{2}^{n} U_{1}^{m}\right|_{\mathcal{H}}$ for all $n, m \geq 0$.

In fact, given $q \in \mathbb{T}$ we can choose $\bar{Q}=Q \oplus q I_{\mathcal{K} \ominus \mathcal{H}}$.

Proof. Fix $q \in \mathbb{T}$. Suppose $\left(\widehat{V}_{2}, \widehat{\mathcal{H}}\right)$ is the minimal unitary dilation of $V_{2}$. Then $\widehat{\mathcal{H}}=\overline{\operatorname{span}}\left\{\widehat{V}_{2}^{n} \mathcal{H}\right.$ : $n \in \mathbb{Z}\}$ and $\widehat{V}_{2}$ is an extension of $V_{2}$. Define $\widehat{V}_{1}: \widehat{\mathcal{H}} \rightarrow \widehat{\mathcal{H}}$ by

$$
\widehat{V}_{1}\left(\widehat{V}_{2}^{n} h\right)=\left(\widehat{Q}^{*} \widehat{V}_{2}\right)^{n} V_{1} h \quad \forall h \in \mathcal{H}, n \in \mathbb{Z},
$$

where $\widehat{Q}=\left(Q \oplus q I_{\widehat{\mathcal{H}} \ominus \mathcal{H}}\right) \in \mathcal{B}(\widehat{\mathcal{H}})$. Then for all $h, h^{\prime} \in \mathcal{H}$ and $n \geq m$,

$$
\begin{aligned}
\left\langle\widehat{V}_{1}\left(\widehat{V}_{2}^{n} h\right), \widehat{V}_{1}\left(\widehat{V}_{2}^{m} h^{\prime}\right)\right\rangle & =\left\langle\left(\widehat{Q}^{*} \widehat{V}_{2}\right)^{n-m} V_{1} h, V_{1} h^{\prime}\right\rangle \\
& =\left\langle\left(\widehat{Q}^{*} \widehat{V}_{2}\right)^{n-m-1} \widehat{Q}^{*} \widehat{V}_{2} V_{1} h, V_{1} h^{\prime}\right\rangle \\
& =\left\langle\left(\widehat{Q}^{*} \widehat{V}_{2}\right)^{n-m-1} Q^{*} V_{2} V_{1} h, V_{1} h^{\prime}\right\rangle \\
& =\left\langle\left(\widehat{Q}^{*} \widehat{V}_{2}\right)^{n-m-1} V_{1} V_{2} h, V_{1} h^{\prime}\right\rangle \\
& =\left\langle V_{1} V_{2}^{n-m} h, V_{1} h^{\prime}\right\rangle \quad \quad \quad(\text { by repeating above steps }) \\
& =\left\langle\widehat{V}_{2}^{n-m} h, h^{\prime}\right\rangle \quad \quad\left(\left.\because \widehat{V}_{2}\right|_{\mathcal{H}}=V_{2} \text { and } n-m \geq 0\right) \\
& =\left\langle\widehat{V}_{2}^{n} h, \widehat{V}_{2}^{m} h^{\prime}\right\rangle .
\end{aligned}
$$

Thus $\widehat{V}_{1}$ is a well defined isometry. Clearly $\widehat{V}_{1}$ is an extension of $V_{1}$. Moreover, $\widehat{Q} \widehat{V}_{1} \widehat{V}_{2}=\widehat{V}_{2} \widehat{V}_{1}$ on $\widehat{\mathcal{H}}$. Suppose $\left(U_{1}, \mathcal{K}\right)$ is the minimal unitary dilation (and hence an extension) of $\widehat{V}_{1}$, so that $\mathcal{K}=\overline{\operatorname{span}}\left\{U_{1}^{n}(\widehat{\mathcal{H}}): n \in \mathbb{Z}\right\}=\overline{\operatorname{span}}\left\{U_{1}^{n}(\widehat{\mathcal{H}}): n \leq 0\right\}$ as $U_{1}$ leaves $\widehat{\mathcal{H}}$ invariant. Define $U_{2}: \mathcal{K} \rightarrow \mathcal{K}$ by

$$
U_{2}\left(U_{1}^{n} \widehat{h}\right)=\left(\bar{Q} U_{1}\right)^{n} \widehat{V}_{2} \widehat{h} \quad \forall \widehat{h} \in \widehat{\mathcal{H}}, n \in \mathbb{Z},
$$

where $\bar{Q}=\left(Q \oplus q I_{\mathcal{K} \ominus \mathcal{H}}\right) \in \mathcal{B}(\mathcal{K})$. As in the case of $\widehat{V}_{1}$, it can be verified that $U_{2}$ is also a well defined isometric extension of $\widehat{V}_{2}$. Clearly $U_{2} U_{1}=\bar{Q} U_{1} U_{2}$. Now we shall prove that $U_{2}$ is onto, so that it is a unitary. For, if $n>0$ let

$$
\mathcal{K}_{n}=\overline{\operatorname{span}}\left\{U_{1}^{* j} \widehat{h}: 0 \leq j \leq n, \widehat{h} \in \widehat{\mathcal{H}}\right\}=\operatorname{span}\left\{U_{1}^{* j} \widehat{h}: 0 \leq j \leq n, \widehat{h} \in \widehat{\mathcal{H}}\right\} .
$$

We prove by induction that $U_{2}$ maps $\mathcal{K}_{n}$ onto $\mathcal{K}_{n}$ for every $n>0$. Suppose $n=1$. Then for all $0 \leq j \leq 1$ and $\widehat{h} \in \widehat{\mathcal{H}}$ we have $U_{1}^{* j} \widehat{V}_{2}^{*} \widehat{Q}^{j} \widehat{h} \in \mathcal{K}_{1}$ and

$$
U_{2}\left(U_{1}^{* j} \widehat{V}_{2}^{*} \widehat{Q}^{j} \widehat{h}\right)=\left(\bar{Q} U_{1}\right)^{* j} \widehat{V}_{2} \widehat{V}_{2}^{*} \widehat{Q}^{j} \widehat{h}=U_{1}^{* j} \bar{Q}^{* j} \widehat{Q}^{j} \widehat{h}=U_{1}^{* j} \widehat{h} .
$$

Thus $U_{2}\left(\mathcal{K}_{1}\right)=\mathcal{K}_{1}$. Now assume that $U_{2}$ maps $\mathcal{K}_{n}$ onto $\mathcal{K}_{n}$. To prove that $U_{2}$ maps $\mathcal{K}_{n+1}$ onto $\mathcal{K}_{n+1}$ it is enough to prove that $U_{1}^{*(n+1)} \widehat{h}$ has a pre-image for every $\widehat{h} \in \widehat{\mathcal{H}}$. Since $U_{1}^{* n} \widehat{h} \in \mathcal{K}_{n}$ there exists $x \in \mathcal{K}_{n}$ such that $U_{2}(x)=U_{1}^{* n} \widehat{h}$. Note that $\mathcal{H} \subseteq \widehat{\mathcal{H}} \subseteq \mathcal{K}_{n} \subseteq \mathcal{K}$, hence $\mathcal{K}_{n}$ is reducing for $\bar{Q}$. Therefore there exists $z \in \mathcal{K}_{n}$ such that $U_{2}(z)=\bar{Q} U_{2} x$. Clearly $U_{1}^{*}(z) \in \mathcal{K}_{n+1}$, and

$$
U_{2} U_{1}^{*} z=U_{1}^{*} \bar{Q}^{*} U_{2}(z)=U_{1}^{*} U_{2} x=U_{1}^{* n+1} \widehat{h} .
$$

Thus $U_{2}$ maps $\mathcal{K}_{n+1}$ onto $\mathcal{K}_{n+1}$. By induction we conclude that $U_{1}^{* n} \widehat{h}$ has a pre-image under $U_{2}$ for all $n>0, \widehat{h} \in \widehat{\mathcal{H}}$. Since $\mathcal{K}=\overline{\operatorname{span}}\left\{U_{1}^{n}(\widehat{\mathcal{H}}): n \leq 0\right\}$ we conclude that $U_{2}$ is onto. Note that $\left(\bar{Q}, U_{1}, U_{2}, \mathcal{K}\right)$ is the required quadruple. 
Corollary 2.19. Let $W_{1}, W_{2} \in \mathcal{B}(\mathcal{H})$ be co-isometries and $Q \in \mathcal{B}(\mathcal{H})$ be a unitary such that $W_{2} W_{1}=W_{1} W_{2} Q$. Then there exist a Hilbert space $\mathcal{K} \supseteq \mathcal{H}$ and unitaries $\bar{Q}, U_{1}, U_{2} \in \mathcal{B}(\mathcal{K})$ such that

(i) $U_{2} U_{1}=U_{1} U_{2} \bar{Q}$; and

(ii) $U_{i}$ is a lifting (and hence a dilation) of $W_{i}$ so that $W_{1}^{n} W_{2}^{m}=\left.P_{\mathcal{H}} U_{1}^{n} U_{2}^{m}\right|_{\mathcal{H}}$ and $W_{2}^{n} W_{1}^{m}=$ $\left.P_{\mathcal{H}} U_{2}^{n} U_{1}^{m}\right|_{\mathcal{H}}$ for all $n, m \geq 0$.

In fact, given $q \in \mathbb{T}$ we can choose $\bar{Q}=Q \oplus q I_{\mathcal{K} \ominus \mathcal{H}}$.

Proof. Since $W_{i}^{*}$ 's are isometries satisfying $W_{2}^{*} W_{1}^{*}=Q W_{1}^{*} W_{2}^{*}$, from above theorem there exists Hilbert space $\mathcal{K} \supseteq \mathcal{H}$ and unitaries $U_{1}, U_{2} \in \mathcal{B}(\mathcal{K})$ such that $U_{i}^{*}$ 's are extensions of $W_{i}^{*}$ 's with $U_{2}^{*} U_{1}^{*}=\left(Q \oplus q I_{\mathcal{H}^{\perp}}\right) U_{1}^{*} U_{2}^{*}$.

Combining Theorems 2.16, 2.18 and Corollaries 2.17, 2.19] we have the following analogue of Ando's theorem for $Q$-commuting contractions.

Theorem 2.20 ( $Q$-commuting unitary dilation). Let $T_{1}, T_{2} \in \mathcal{B}(\mathcal{H})$ be contractions and $Q \in \mathcal{B}(\mathcal{H})$ be a unitary such that $T_{2} T_{1}=Q T_{1} T_{2}$ (respectively $T_{2} T_{1}=T_{1} T_{2} Q$ ). Then there exist Hilbert space $\mathcal{K} \supseteq \mathcal{H}$ and unitaries $\bar{Q}, U_{1}, U_{2} \in \mathcal{B}(\mathcal{K})$ such that

(i) $U_{2} U_{1}=\bar{Q} U_{1} U_{2}$ (respectively $\left.U_{2} U_{1}=U_{1} U_{2} \bar{Q}\right)$; and

(ii) $T_{1}^{n} T_{2}^{m}=\left.P_{\mathcal{H}} U_{1}^{n} U_{2}^{m}\right|_{\mathcal{H}}$ and $T_{2}^{n} T_{1}^{m}=\left.P_{\mathcal{H}} U_{2}^{n} U_{1}^{m}\right|_{\mathcal{H}}$ for all $n, m \geq 0$.

In fact, given $q \in \mathbb{T}$ we can choose $\bar{Q}=Q \oplus q I_{\mathcal{K} \ominus \mathcal{H}}$.

Remark 2.21. If $Q=q I_{\mathcal{H}}$ for some $q \in \mathbb{T}$, then Theorem 2.20 reduces to $q$-commuting dilation theorem.

Acknowledgment. The first author thanks the Department of Atomic Energy (DAE), Government of India for financial support and IMSc Chennai for providing necessary facilities to carry out this work. We thank B. V. R. Bhat for helpful suggestions.

\section{REFERENCES}

[1] T. Ando, On a pair of commutative contractions, Acta Sci. Math. (Szeged) 24 (1963), 88-90.

[2] R. G. Douglas, P. S. Muhly And C. Pearcy, Lifting commuting operators, Michigan Math. J. 15 (1968), 385-395.

[3] T. Iто, On the commutative family of subnormal operators, J. Fac. Sci. Hokkaido Univ. Ser. I 14 (1958), $1-15$.

[4] D. K. Keshari and N. Mallick, q-commuting dilation, Proc. Amer. Math. Soc. 147 (2019), no. 2, 655-669.

[5] B. Sz.-NAGY, Sur les contractions de l'espace de Hilbert, Acta Sci. Math. Szeged 15 (1953), 87-92.

[6] B. Sz.-Nagy and C. Foias, Dilatation des commutants d'opérateurs, C. R. Acad. Sci. Paris Sér. A-B 266 (1968), A493-A495. MR0236755

[7] B. Sz.-Nagy And C. Folas, Harmonic analysis of operators on Hilbert space, Translated from the French and revised, North-Holland Publishing Co., Amsterdam, 1970.

[8] Z. Sebestyen, Anticommutant lifting and anticommuting dilation, Proc. Amer. Math. Soc. 121 (1994), no. $1,133-136$.

[9] J. J. Schaffer, On unitary dilations of contractions, Proc. Amer. Math. Soc. 6 (1955), 322. 
[10] Z. Sebestyen, Lifting intertwining operators, Period. Math. Hungar. 28 (1994), no. 3, 235-240. 